\title{
Editorial: Indian Guidelines for Treatment of Pediatric Malignancies
}

\author{
Akash Tiwari $^{1} \cdot$ Sameer Bakhshi $^{1}$ \\ Received: 1 March 2017 / Accepted: 1 March 2017 /Published online: 16 March 2017 \\ (C) Dr. K C Chaudhuri Foundation 2017
}

Treatment strategies and protocols for pediatric tumors vary by health provider, treating center and geographical area within the country. The problem has been compounded by the rapid evolution in the world of oncology and advent of new technologies and treatment options. The cost of health care has also increased manifold in the recent times; further, all treatment options and modalities of diagnosis are not equally available in all places. This urges a need for a national guideline for management of pediatric tumors, which are made by experts in the field and with an Indian perspective in mind.

These guidelines have been made with intent to bridge the gap between evidence and practice in the country, and make patient care more consistent. A multidisciplinary team of experts has contributed to the development of these guidelines. These recommendations are based on a detailed review of available Indian and international literature, and expert opinion has been used where adequate evidence was lacking. These guidelines cover the common pediatric tumors, including Hodgkin lymphoma (HL) and non-Hodgkin lymphoma (NHL), rhabdomyosarcoma, Wilms tumor, neuroblastoma, hepatoblastoma and germ cell tumor [1-7]. The prime focus of these guidelines includes diagnostic workup, and medical and surgical management of pediatric tumors. The guidelines are largely consistent with the pediatric oncology guidelines by other international bodies.

The review is complete and includes the most-recent abstracts and articles. For example, the guidelines have accepted the results of the interim analysis of Inter-B-NHL Ritux 2010

Sameer Bakhshi

sambakh@hotmail.com

1 Department of Medical Oncology, Dr. B.R.A Institute-Rotary Cancer Hospital, All India Institute of Medical Sciences, New Delhi 110029, India trial and recommend the use of rituximab in high-risk B-NHL along with intensive chemotherapy protocol [8]; use of rituximab is also recommended in patients with relapsed B-NHL.

The prime benefit of having an evidence-based guideline is that it helps patients from across the country to get standard, evidence-based uniform care. This will ensure that the quality of care is not compromised based on where and by whom the patient is being treated, and is likely to improve the outcomes of these patients and decrease the disease-related morbidity and mortality. The cost-effectiveness of management of these tumors is also likely to improve by eliminating unnecessary diagnostic evaluations and treatment that add to cost but are not based on evidence. Few examples of such practices are use of fine needle aspiration cytology (FNAC) for diagnosis of lymphomas and frequent use of imaging in the follow-up of these patients. The guidelines on HL specifically discourage use of FNAC for diagnosis and also limit imaging studies in follow-up period [1]. This will sensitize local practitioners and small centers to move away from these practices.

These guidelines also address some of the ground realities of the country; for example, it stresses upon the fact that neoadjuvant chemotherapy followed by surgery is the preferred approach in children with Wilms tumors, keeping in mind the size of the tumors at presentation, and the likely difficulties faced in upfront resection [4]. At the same time, the guidelines recognise the fact that the SIOP protocol for Wilms tumor would be difficult to implement as is used in Europe, because of the scarcity of pathologists who are trained and experienced in pediatric renal tumors. Another benefit of these guidelines is that their implementation is likely to facilitate the collaborative work between various centers as more patients would be on uniform protocols.

A major pitfall of these guidelines is over-reliance on international literature in a want of data from our country. Thus, if a problem is indigenous to our country, we have to depend on 
expert opinions more than evidence. These guidelines highlight the paucity of prospective data in pediatric tumors from our country. The studies from India are largely retrospective and are in the form of a review of institutional data [9]. This limited data is from select tertiary care centers within the country which may not fully reflect the outcomes across a diverse country like India with variable standards of care.

Further, there are some tumors such as rhabdomyosarcoma wherein there is no published prospective or retrospective peer-reviewed data on outcome from India. Some of the unpublished data presented in Indian meetings suggest that the outcome of patients with rhabdomyosarcoma is much inferior to that seen in the Western world. In the absence of any published articles, there are no well-defined reasons for these poorer outcomes. These guidelines do not address this discrepancy in the outcome, and neither do they suggest any interventions to improve the same.

The review of literature in the guidelines also brings out the lack of published multicentric data from India. Recently, multicentric data on HL was published from Delhi suggesting that this is indeed possible [10]. Furthermore, like any other guidelines, the implementation of these guidelines and adapting it to the local needs and resources would remain a major hurdle in a country like India where resources and availability of health care facilities are so diverse.

We need to design strategies to ensure appropriate dissemination and implementation of these guidelines at a national level. Once implemented, a mechanism needs to be devised for proper evaluation of the impact of these guidelines on all aspects of patient care and challenges faced in its implementation. These guidelines must be revised at fixed intervals to ensure that the recommendations of these guidelines are in accordance with the recent scientific evidence, and that they are not outdated. The further versions of these guidelines should build upon the experiences of those who have contributed in making these guidelines and continuous feedback from the users. This will ensure better implementation of these guidelines and their adaptation to local needs and resources.

The future of medical research in India lies in collaboration between various institutes. A major step forward has been taken in this direction by the constitution of the Indian Pediatric Oncology Group (InPOG) [11]. The implementation of these guidelines will facilitate a uniform treatment protocol across the country and will further strengthen this initiative. It is likely that these steps will contribute towards the strengthening of research in India in the field of pediatric oncology.

\section{Compliance with Ethical Standards}

Conflict of Interest None.

Source of Funding None.

\section{References}

1. Radhakrishnan V, Kapoor G, Arora B, et al. Management of Hodgkins lymphoma: ICMR consensus document. Indian J Pediatr. 2017; doi:10.1007/s12098-017-2304-6.

2. Thacker N, Bakhshi S, Chinnaswamy G, et al. Management of nonHodgkin lymphoma: ICMR consensus document. Indian J Pediatr. 2017; doi:10.1007/s12098-017-2318-0.

3. Panda SP, Chinnaswamy G, Vora T, et al. Diagnosis and management of rhabdomyosarcoma in children and adolescents: ICMR consensus document. Indian J Pediatr. 2017; doi:10.1007/s12098017-2315-3.

4. Prasad M, Vora T, Agarwala S, et al. Management of Wilms tumor: ICMR consensus document. Indian J Pediatr. 2017; doi:10.1007 /s12098-017-2305-5.

5. Bansal D, Totadri S, Chinnaswamy G, et al. Management of neuroblastoma: ICMR consensus document. Indian J Pediatr. 2017; doi:10.1007/s12098-017-2298-0.

6. Agarwala S, Gupta A, Bansal D, et al. Management of hepatoblastoma: ICMR consensus document. Indian J Pediatr. 2017; doi:10.1007/s12098-017-2301-9.

7. Agarwala S, Mitra A, Bansal D, et al. Management of pediatric malignant germ cell tumors: ICMR consensus document. Indian J Pediatr. 2017; doi:10.1007/s12098-017-2308-2.

8. Minard-Colin V, Auperin A, Pillon M, et al. Results of the randomized intergroup trial Inter-B-NHL Ritux 2010 for children and adolescents with high-risk B-cell non-Hodgkin lymphoma (B-NHL) and mature acute leukemia (B-AL): evaluation of rituximab $(\mathrm{R})$ efficacy in addition to standard LMB chemotherapy $(\mathrm{CT})$ regimen. [Abstract] J Clin Oncol. 2016;34:A-10507.

9. Agarwala S, Mandelia A, Bakhshi S, et al. Neuroblastoma: outcome over a 14 year period from a tertiary care referral centre in India. J Pediatr Surg. 2014;49:1280-5.

10. Bhethanabhotla S, Jain S, Kapoor G, et al. Outcome of pediatric advanced Hodgkin lymphoma treated with ABVD and predictors of inferior survival: a multicenter study of 186 patients. Leuk Lymphoma. 2016;1-7

11. Arora RS, Bakhshi S. Indian pediatric oncology group (InPOG) collaborative research in India comes of age. Pediatr Hematol Oncol J. 2016;1:13-7. 